\title{
Health care suggestions for COVID 19 pandemic crisis
}

\author{
Vivek Kamath*
}

Heal the World Organization, India

\section{Communication}

1. Educate key health care professionals working in hospital about COVID 19 Disease, SARS and MERS as all these corona viruses are a large family of enveloped RNA Viruses that mostly infect birds and mammals. How these diseases are different from Covid 19? What have we done to overcome SARS and MERS diseases? Brief all of them on function, anatomy of respiratory system.

2. Share the accurate statistics of the COVID 19 deaths, fatality rate of COVID19 till date across the globe and India

3. Train all the health care professionals how to combat virus infection from prevention state to till end state of infections.

4. It is important that all hospital should prepare check list to build strong immune system.

a. All health care professionals need to undergo immunity test (Immunoglobin Test) before working in hospital

b. If they have any critical ailment or illness, they should not be allowed to work in hospital till they recover from it

c. They should be trained in understanding our body immune system and how it can combat virus attacks

d. A check list to build a robust immune system needs to be prepared and all health care professional should follow it on daily basis

e. Hospital Management team should publish this and circulate to all patients

f. Hospital management team should not pressurize health care professional to work over time. All health care professionals should take enough rest, sleep and nutritious food.

g. All health care professionals, patients need to stop smoking, alcohol and drugs to safe guard their immune system

h. Hospital should hire expert health counsellors, psychology expert/ mind counselling experts to brief health care professionals and try to remove or manage the fear, stress, phobia or panic attacks about COVID 19 attack from their subconscious mind. The same procedure needs to be followed to brief all COVID 19 patients.

i. A good nutrition and other alternative healing therapies are the keys to followed by health care professional and patients during the treatments

Copyright: (C2020 Kamath V. This is an open-access article distributed under the terms of the Creative Commons Attribution License, which permits unrestricted use, distribution, and reproduction in any medium, provided the original author and source are credited. j. Health care professional needs to get trained in alternative therapies like Reiki, Meditation, Crystal Healing, Mexican healing, Melchizedek healing, Yoga, Pranic Healing and mind healing techniques to take care of their body and mind challenges

5. Hospital management needs to document all prevailing diseases of the patients carefully and group the patients based on 2 categories of the diseases for e.g. 1. COVID 19 case no other disease history 2. COVID 19 Case with prevailing disease (if It is cancer patient highlight it in the document)

6. After the patient recovery, doctors and hospital authority should document the treatment given to patients, time to recovery in every stage of the diseases, ventilator usages and issues faced, etc.

7. In case of death due to covid 19 cases, document all the complications faced by patients in every stage of the treatment and reason behind the failure of the treatment. Any specific technical issues like ventilator problems needs to be documented clearly. Do they really need ventilators or able to substitute with simpler or widely available devices? As per the research report, unusually high death rates of covid 19 patients on ventilators observed.

As per medical experts, 40 to $50 \%$ of patients with severe respiratory distress die while on ventilators. However, this percentage varied from country to country. New York city showed $80 \%$ death on ventilators, Wuhan showed it as $86 \%$ and UK reported it as $66 \%$.

There could be possibility that high-pressure oxygen forced in tiny air sacs into patient's lungs. This major issue needs to be discussed and addressed by health care experts across the globe immediately before putting elderly patients on ventilators.

8. People needs to be honest when they fall sick or have any respiratory problems, flu symptoms etc. to call up the health authority/hospitals and register themselves.

9. It is important to note that people who do not have any health issues not required to wear masks all the time. It may be required to wear, when you go near infected person. People who are suffering from sneezing, coughing, cold, flu, etc. need to wear the masks to avoid spreading the viruses.

10.Social distancing can be followed as per the guideline provided by health care authority

${ }^{\star}$ Correspondence to: Vivek Kamath, Heal the World Organization, India, Tel: +917760749867, E-mail: vivek.kamath@healtheworld.in

Received: Mar 05, 2020; Accepted: May 12, 2020; Published: May 19, 2020 